\section{Palliative care}

\section{SUPPORTIVE FUNCTION OF PEGTEOGRASTIM AND PEGFILGRASTIM ON CHEMOTHERAPY-INDUCED NEUTROPENIA IN OVARY CANCER PATIENTS}

Min Kyu Kim. Sungkyunkwan University of Medicine,Samsung Changwon Hospital

\subsection{6/ijgc-2020-ESG0.153}

Introduction/Background Critical complication during chemotherapy is febrile neutropenia.. Granulocyte-colony-stimulating factor(G-CSF) is used to prevent febrile neutropenia associated with myelosuppression. Pegfilgrastim, a pegylated form of filgrastim, has an increased half-life. Pegteograstim is novel recombination human G-CSF of another form of pegylated filgrastim. We undertook investigation to evaluate efficacy and safety of pegteograstim and pegfilgrastim women with ovarian carcinoma that are treated with paclitaxel/carboplatin.

Methodology After chemotherapy minimum 24 hours, pegteograstim or pegfilgrastim was given a single subcutaneous injection of $6 \mathrm{mg}$ during each chemotherapy cycle. We evaluated to ANC (absolute neutrophil count) change and febrile neutropenia incidence.

Results There were 30 of pegteograstim cases and 12 pegfilgrastim. Median ANC between pegteostim were 2960.pegfilgrastim was 2396.After pegteograstim, ANC was elevated till 13847 from 2960 (difference was 10,887)in case of pegteograstim. In pegfilgrastim, ANC increased to 12933 (difference was 10537).There was no febrile neutropenia in both cases. Safety profiles of two groups did not differ significantly.

Conclusion We conclude Pegteograstim and pegfilgrastim have similar efficacy and safety profile in the reduction of chemotherapy-induced neutropenia in the ovary cancer patients who were undergoing chemotherapy.

Disclosures NO COI.

\section{EFFICACY OF INDIVIDUALISED STARTING DOSE (ISD) AND FIXED STARTING DOSE (FSD) OF NIRAPARIB PER INVESTIGATOR ASSESSMENT (IA) IN NEWLY DIAGNOSED ADVANCED OVARIAN CANCER (OC) PATIENTS}

${ }^{1}$ Ulla Peen, ${ }^{2}$ Whitney Graybill, ${ }^{3}$ Antonio González-Martin, ${ }^{4}$ David O'malley, ${ }^{5} J$ eanFrançois Baurain, ${ }^{6}$ Emily Prendergast, ${ }^{7}$ Philippe Follana, ${ }^{8}$ Elena I Braicu, ${ }^{9}$ Divya Gupta, ${ }^{10}$ Bradley J Monk. 'Herlev University Hospital; ${ }^{2}$ Gynecologic Oncology Group (Gog); Medical University of South Carolina; Department of Gynecologic Oncology; ${ }^{3}$ Grupo Español de Investigación En Cáncer de Ovario (Geico); Clínica Universidad de Navarra; Medical Oncology Department; ${ }^{4}$ Ohio State University - James Comprehensive Cancer Center; ${ }^{5}$ Cliniques Universitaires Saint-Luc, Université Catholique de Louvain; ${ }^{6}$ Minnesota Oncology; ${ }^{7}$ Gineco; Centre Antoine Lacassagne; ${ }^{8}$ Charité Medical University; ${ }^{9}$ Glaxosmithkline; ${ }^{10}$ Arizona Oncology (US Oncology Network), University of Arizona College of Medicine

\subsection{6/ijgc-2020-ESG0.154}

Introduction Niraparib is a poly(ADP-ribose) polymerase inhibitor approved for maintenance treatment of patients with newly diagnosed or recurrent OC that responded to platinumbased chemotherapy and treatment in heavily-pretreated recurrent OC. Here we report efficacy in patients receiving the FSD and ISD in the PRIMA/ENGOT-OV26/GOG-3012 trial (NCT02655016).

Methods This double-blind, placebo-controlled, phase 3 study randomised 733 patients to receive niraparib or placebo for 36 months or until disease progression/toxicity. A protocol amendment introduced ISD: $200 \mathrm{mg}$ in patients with body weight $<77 \mathrm{~kg}$ or platelets $<150,000 / \mu \mathrm{L}$, or $300 \mathrm{mg}$ in all others. The primary endpoint was PFS by blinded independent central review (BICR). IA PFS was a sensitivity analysis. At the primary analysis data cut, follow-up was 11.2 months and 17.1 months in the ISD and FSD subgroups, respectively. An ad hoc analysis of IA PFS was performed using an updated data cut with additional 6 months follow-up.

Results BICR and IA PFS were highly concordant in the overall population. Efficacy of niraparib based on IA PFS in FSD vs ISD subgroups for each data cut were similar (table 1). Dose interruptions, modifications, and haematologic toxicity were lower with the ISD. Exposure-response data supported the clinical data.

Conclusion The 200- or 300-mg ISD by baseline body weight and platelet counts demonstrated comparable efficacy while improving the safety profile of niraparib. Use of this regimen for first-line maintenance of advanced OC patients is approved by the US FDA.

Disclosures Funded by: GlaxoSmithKline

NCT: NCT02655016

Encore statement: This data is presented on behalf of the original authors with their permission. Presented at the International Gynecologic Cancer Society (IGCS) Annual Global Meeting, September 10-13, 2020, Virtual.

Dr. González-Martín reports personal fees and non-financial support from AstraZeneca; Grant and personal fees from GSK, Clovis Oncology, Roche Holding AG, Merck \& Co., Inc., Genmab, INMUNOGEN, Pharma Mar, S.A., and Oncoinvent AS.

Dr. Graybill reports personal fees from GSK.

Dr. O'Malley reports personal fees from Immunogen, Eisai, Agenus, GSK : Consultant/Advisory Board for Clovis, Ambry, Abbvie, Janssen/J\&J, Regeneron, Novacure, Myraid Genetics, Tarveda, Amgen, VentiRx, Array Biopharma, EMD Serono, Ergomed; Steering committee for Genentech/Roche and Merck; Institutional funding from Ajinomoto Inc, Ludwig Cancer Research, Stemcentrx, Inc, CERULEAN PHARMA, GOG Foundation, BMS, Serono Inc, TRACON Pharmaceuticals, Yale University, New Mexico Cancer Care Alliance, INC Research, Inc., Inventiv Health Clinical, Iovance Biotherapeutics, Inc, and PRA International.

Dr. Monk reports consulting and advisory role at Merck, GSK, Roche/Genentech, AstraZeneca, Advaxiz, Cerulean Pharma, Amgen, Immunogen, NuCana BioMed, Clovis Oncology, Pfizer, Mateon Therapeutics, Precision Oncology, Perthera, Abbvie, Myriad Pharmaceuticals, Incyte, VBL Therapeutics, Takeda, Samumed, Oncomed, OncoSec, ChemoID,

\section{Abstract 388 Table 1}

\begin{tabular}{|c|c|c|}
\hline \multirow[b]{2}{*}{ Median PFS } & \multicolumn{2}{|c|}{$\begin{array}{c}\text { IA PFS in the overall population and the ISD and FSD } \\
\text { subgroups, } \mathrm{HR}(95 \% \mathrm{Cl})\end{array}$} \\
\hline & $\begin{array}{l}\text { Original data cut } \\
17 \text { May } 2019\end{array}$ & $\begin{array}{l}\text { Updated data cut } \\
17 \text { Nov } 2019 \\
\end{array}$ \\
\hline Overall, N=733 & $\begin{array}{l}0.63 \\
(0.51,0.76) \\
P<0.0001\end{array}$ & $\begin{array}{l}0.64 \\
(0.53,0.77) \\
P<0.0001\end{array}$ \\
\hline FSD, $n=487$ & $\begin{array}{l}0.60 \\
(0.47,0.77)\end{array}$ & $\begin{array}{l}0.62 \\
(0.49,0.78)\end{array}$ \\
\hline ISD, n=246 & $\begin{array}{l}0.68 \\
(0.48,0.96)\end{array}$ & $\begin{array}{l}0.68 \\
(0.49,0.94)\end{array}$ \\
\hline
\end{tabular}

FSD, fixed starting dose; HR, hazard ratio; IA, investigator assessment; ISD, individualised starting dose; PFS, progression-free survival. 